\title{
Correction to: Application of PET and SPECT to the Study of Autism Spectrum Disorders
}

Diane C. Chugani and Samira Mukarram

\section{Correction to: R. A. J. O. Dierckx et al. (eds.), PET and SPECT in Psychiatry, https://doi.org/10.1007/978-3-030-57231-0_29}

The book was inadvertently published with an incorrect spelling of the author's surname in Chapter 29 as Mukarran whereas it should be Mukarram. This error has now been corrected with this erratum. 